\title{
Enhanced-throughput production of polymersomes using a parallelized capillary microfluidic device
}

\author{
Shin-Hyun Kim • Jin Woong Kim • Do-Hoon Kim • \\ Sang-Hoon Han · David A. Weitz
}

Received: 18 July 2012/Accepted: 4 September 2012/Published online: 13 October 2012

(C) Springer-Verlag Berlin Heidelberg 2012

\begin{abstract}
We report a parallelized capillary microfluidic device for enhanced production rate of monodisperse polymersomes. This device consists of four independent capillary microfluidic devices, operated in parallel; each device produces monodisperse water-in-oil-in-water $(\mathrm{W} / \mathrm{O} / \mathrm{W})$ doubleemulsion drops through a single-step emulsification. During generation of the double-emulsion drops, the innermost water drop is formed first and it triggers a breakup of the middle oil phase over wide range of flow rates; this enables robust and stable formation of the double-emulsion drops in all drop makers of the parallelized device. Double-emulsion drops are transformed to polymersomes through a dewetting of the amphiphile-laden middle oil phase on the surface of the innermost water drop, followed by the subsequent separation of the oil drop. Therefore, we can make polymersomes with a production rate enhanced by a factor given by the number of drop makers in the parallelized device.
\end{abstract}

Keywords Microfluidics · Polymersomes · Emulsions · Microcapsules · Parallelization

\footnotetext{
S.-H. Kim · D. A. Weitz $(\bowtie)$

Department of Physics and School of Engineering and Applied

Sciences, Harvard University, Cambridge, MA, USA

e-mail: weitz@seas.harvard.edu

S.-H. Kim $(\bowtie)$

Department of Chemical and Biomolecular Engineering,

KAIST, Daejeon, South Korea

e-mail: kim.sh@kaist.ac.kr

J. W. Kim

Department of Applied Chemistry, Hanyang University,

Ansan, South Korea

D.-H. Kim · S.-H. Han

Amore-Pacific Co. R\&D Center, Yongin, South Korea
}

\section{Introduction}

Emulsion drops can be utilized as templates to make microparticles and capsules. To achieve high uniformity in the size and functionality of these structures, microfluidic devices, fabricated either with poly(dimethylsiloxane) or glass capillaries, can be used to produce emulsion droplets, thereby providing a high degree of controllability on size, shape, and properties of the drops (Anna et al. 2003; Thorsen et al. 2001; Utada et al. 2005; Kim and Weitz 2011). For example, single-emulsion drops, prepared in such microfluidic devices, can be employed to make monodisperse spherical microparticles with optical, electrical, and magnetic functionalities (Kim et al. 2008a, 2010; Nie et al. 2006; Nisisako et al. 2006; Zhao et al. 2008). In addition, nonspherical microparticles can also be prepared by deforming the drops or making pairs of drops (Dendukuri et al. 2005; Hwang et al. 2008; Kim et al. 2011a; Nisisako and Torii 2007; Xu et al. 2005). Moreover, microfluidic emulsification enables generation of doubleemulsion drops, or drops in drops, in an efficient way, which is otherwise difficult to achieve using bulk emulsification (Okushima et al. 2004; Utada et al. 2005). Such doubleemulsion drops can be used as templates to create microcapsules with a core-shell geometry, enabling creation of polymeric microcapsules, vesicles, and colloidosomes (Choi et al. 2009; Kim et al. 2007, 2008b, 2011b; Lee and Weitz 2008; Lorenceau et al. 2005; Shum et al. 2008).

Although such microfluidic emulsification provides highly useful templates for the production of functional microparticles and capsules, there remains intrinsic obstacle to mass production: generation on single drop basis limits production rate. One method to enhance the production rate of emulsion drops or capsules is through parallelization of the emulsification junctions in polymeric 
devices (Nisisako and Torii 2008; Ota et al. 2009; Romanowsky et al. 2012); however, these devices exhibit poor resistance to organic solvents, severely limiting their utility. Moreover, parallelization of double-emulsion makers is much more difficult than that of single-emulsion makers because formation of double-emulsion drops in the device frequently requires chemical patterning and precise control of flow rates. This limitation will be overcome by parallelizing capillary microfluidic devices which exhibit high resistance to chemicals; however, this would require a device with high degree of flow control to ensure that all components operate simultaneously.

In this paper, we report a practical approach to achieve such a parallelized capillary device to make monodisperse double-emulsion drops which can be used as templates to produce polymersomes. Our device consists of four independent capillary devices which simultaneously produce double emulsions; they can be easily operated in parallel through the use of controlled double emulsification: during generation of water-in-oil-in-water (W/O/W) double emulsions, the innermost water drops are formed first and they trigger the breakup of the middle oil phase. This operates over a wide range of flow rates of the innermost, middle, and continuous phases, thereby enabling stable production of the double-emulsion drops in each drop makers in the parallelized device, though the flow rates in each drop maker are slightly different from one another. The resultant double-emulson drops exhibit dewetting of the middle oil phase on the surface of the innermost water drops, followed by subsequent separation of the oil, creating polymersomes whose membranes are composed of a bilayer of amphiphilic diblock-copolymers (Shum et al. 2011; Kim et al. 2011c).

\section{Results and discussion}

\subsection{Device fabrication}

We use glass capillary devices comprising of two tapered circular capillaries (World Precision Instruments, Inc., 1B100-6) inserted in a second square capillary (AIT glass) as schematically illustrated in Fig. 1a (Utada et al. 2005). The inner dimension of the square capillary is very slightly larger than that of the outer diameter of the circular capillaries which is $1 \mathrm{~mm}$; this facilitates alignment of two circular capillaries. One circular capillary is tapered to have small orifice with an outer diameter of $20 \mu \mathrm{m}$ and is coated with n-octadecyltrimethoxyl silane (Aldrich) to make the capillary wall hydrophobic. This is used for injection of the innermost aqueous phase. The other one is tapered to have a larger orifice with an inner diameter of $125 \mu \mathrm{m}$ and is coated with 2-[methoxy(polyethyleneoxy)propyl] (a)

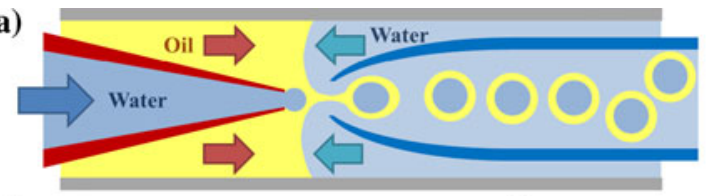

(b)

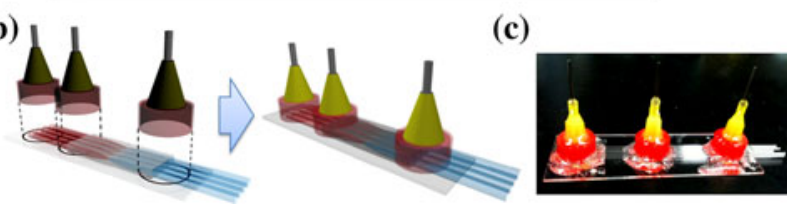

Fig. 1 a Schematic illustration of the microfluidic capillary device for preparation of water-in-oil-in-water $(\mathrm{W} / \mathrm{O} / \mathrm{W})$ double-emulsion drops. The treatment of the surfaces of the capillaries is denoted by different colors, with red signifying hydrophobic surface and blue signifying hydrophilic surface, respectively. b Schematic illustration and $\mathbf{c}$ image of a parallelized device (color figure online)

trimethoxyl silane (Gelest, Inc.) to make the capillary wall hydrophilic. This is used for collection of the doubleemulsion drops. To parallelize this double-emulsion drop maker, we use four square capillaries with the same length and fix them on a glass slide side by side without a gap. The injection and collection capillaries are inserted in the two outer square capillaries and coaxially aligned with the assistance of a microscope and fixed with an epoxy resin. Subsequently, the two inner devices are assembled in the same fashion. To inject fluids into the four different channels simultaneously, the four inlets of the injection capillaries and the square capillaries are covered by a single needle with a wide opening as illustrated schematically in Fig. 1b. The needles (McMaster-CARR, 75165A677) are connected with a top-cut plug-type enclosure (VWR, 211-0042) to attain a wide opening. The openings are patterned to fit into the capillaries and are sealed with epoxy resin, resulting in the parallelized device shown in Fig. 1b, c.

\subsection{Influence of flow rates on generation} of double-emulsion drops

When several independent capillary devices are used in parallel to make double emulsions, there is inevitably some variation in flow rate from device to device. Therefore, to investigate the influence of the flow rate on the generation of double-emulsion drops, we use a single capillary device. We inject a $10 \mathrm{wt} \%$ aqueous solution of poly (ethylene glycol) (PEG, Mw 6,000, Sigma-Aldrich) through the injection capillary, forming the innermost drops. We use a mixture of chloroform and hexane, at a volume ratio of 38:62, containing PEG (Mw 5,000)-b-poly(lactic acid) (PLA, Mw 10,000) diblock-copolymer (Polysciences, Inc.) at a concentration of $5 \mathrm{mg} / \mathrm{ml}$; this forms the middle oil layer and is injected through the interstices between the injection and square capillaries. The continuous phase is 10 
wt $\%$ aqueous solution of poly (vinyl alcohol) (PVA, Mw 13,000-23,000, Sigma-Aldrich); it is injected through the interstices between the collection and square capillaries, as shown in Fig. 1a. Because of the hydrophobic nature of the injection capillary, the innermost aqueous phase flows through the tip of the injection capillary without wetting its outer surface, while the middle oil phase flows along the outer wall of the capillary, coaxially shielding the innermost stream. In addition, the hydrophilic surface of the collection capillary ensures that the middle phase flows through the center of the orifice of the collection capillary without contacting the wall; the continuous phase flows along the inner and outer walls of the collection capillary.

The flow rates of the innermost $\left(Q_{1}\right)$, middle $\left(Q_{2}\right)$, and continuous $\left(Q_{3}\right)$ phases each influence the breakup of two coaxial interfaces, one consisting of the innermost water and middle oil phases and the second consisting of the middle oil and continuous water phases. In a wide range of $Q_{1}$ and $Q_{2}$, the innermost drops are formed first and they then trigger breakup of the middle phase to form the outer drop; this results in the formation of monodisperse doubleemulsion drops, as shown in Fig. 2a, b. We summarize the effects of variation in $Q_{1}$ and $Q_{2}$ at a constant value of $Q_{3}=3,000 \mu \mathrm{l} / \mathrm{h}$, where circles denote the generation of double-emulsion drops without formation of satellite drops, whereas squares and triangles denote the generation of double-emulsion drops with concurrent generation of satellite drops of oil. The diameter of the satellites is much smaller than the double-emulsion drop itself in the case of squares, whereas it is larger than half the diameter of the double-emulsion drops in the case of triangles, as shown in Fig. 2. In this breakup mode, the innermost drops grow at the tip of the injection capillary and are injected into the middle phase when they become comparable in size to the diameter of the orifice of the collection capillary. During formation of these drops, they completely prevent any additional flow of the middle phase; this lead to elongation of the middle phase, resulting in its breakup at its trailing edge of the resultant short jet of the middle fluid. The series of still shot images shows this innermost-drop-triggered breakup in Fig. 3a, where white arrows indicate the growth of the innermost drops and paired arrows with red and blue colors indicate the breakup of trailing edge of outer jet and breakup of the innermost drop, respectively. The resultant double-emulsion drops are highly monodisperse and are comparable in size to the diameter of the orifice. When either $Q_{1}$ or $Q_{2}$ is relatively small, the breakup of the middle phase jet first occurs at its leading edge; this is followed by subsequent breakup at the trailing edge upon injection of the innermost drops. This produces satellite drops, as denoted with squares and triangles in Fig. 2a. The series of still shot images shows this formation of satellite drops in the innermost-drop-triggered breakup mode in

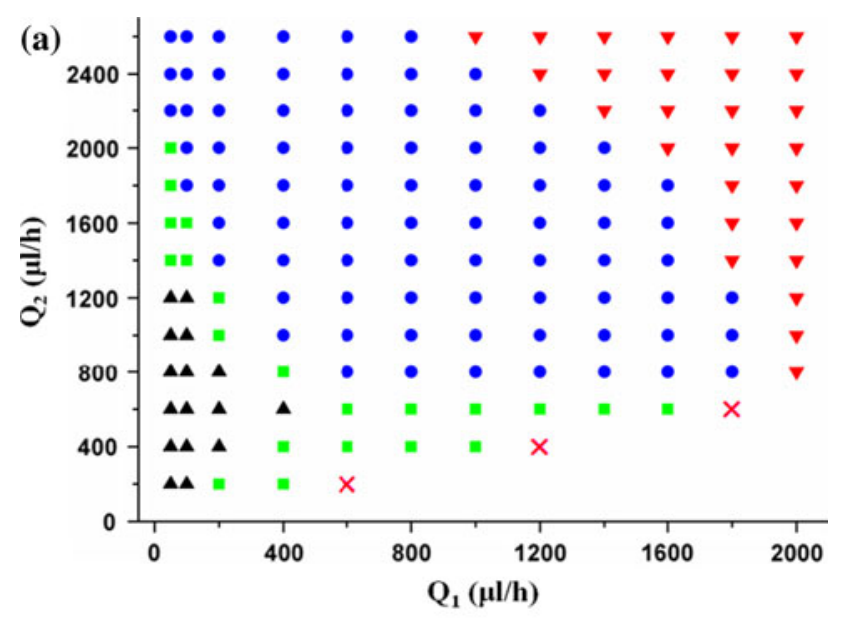

(b)

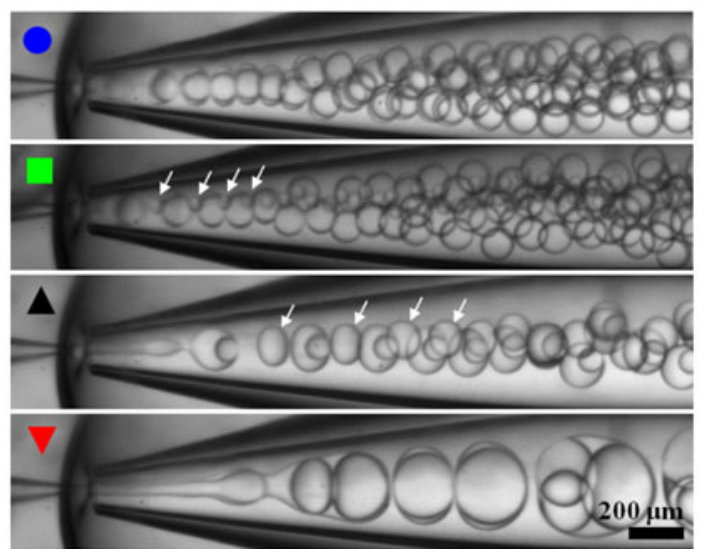

Fig. 2 Flow behavior as a function of volumetric flow rates of innermost $\left(Q_{1}\right)$ and middle $\left(Q_{2}\right)$ phases, where volumetric flow rate of continuous phase $\left(Q_{3}\right)$ is maintained at $3,000 \mu \mathrm{l} / \mathrm{h}$. Blue circles, green squares, and black triangles denote generation of monodisperse doubleemulsion without satellite drops, with small satellite drops, and with large satellite drops, respectively. The satellite drops are denoted with white arrows in $\mathbf{b}$. Red inverted triangles and crosses denote formation of polydisperse double-emulsion drops from a widening jet and a failure of double-emulsion generation, respectively (color figure online)

Fig. 3b, where white arrows indicate the growth of the innermost drops and paired arrows with green and red colors indicate the breakup at the leading edge and subsequent breakup at the trailing edge of the outer jet, respectively. We attribute this first breakup at the leading edge of the jet to the drag force exerted on the jet by the continuous phase. For small $Q_{1}$, the injection frequency of the innermost drops is also low, resulting in a longer jet of the middle phase; therefore, the breakup of the jet at the leading edge occurs prior to the breakup at the trailing edge. For small $Q_{2}$, the difference in linear velocities of the middle and continuous phases is relatively large, resulting in significant drag force on the jet, leading to breakup at the leading edge. All double-emulsion drops produced in these regimes (circles, squares, and triangles), which we denote as innermost-drop-triggered breakup, can be used for 
(a)

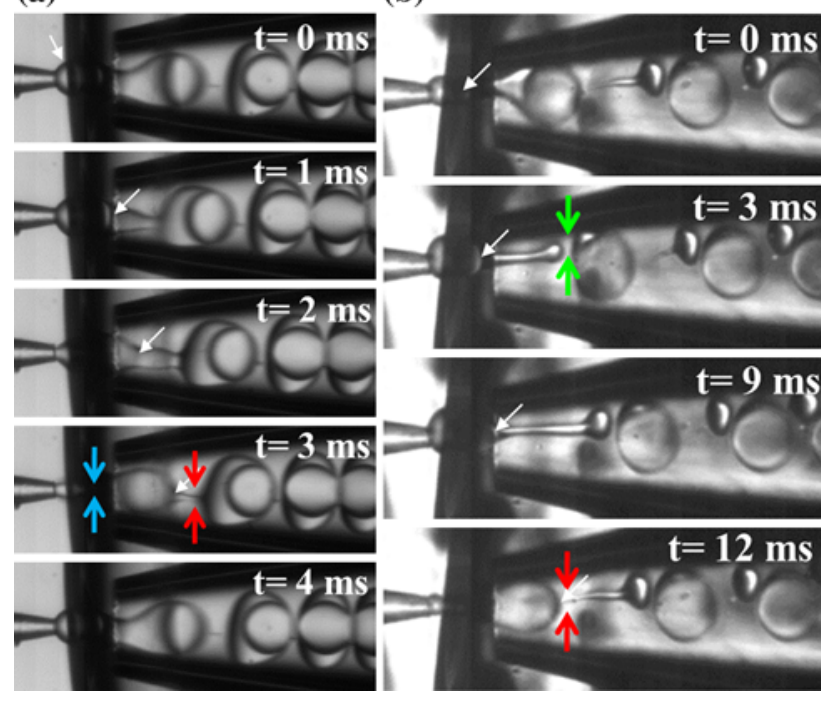

Fig. 3 Series of still shot images showing a the innermost-droptriggered breakup and $\mathbf{b}$ formation of satellite drops in the same breakup mode, taken at the donated time in the images. White arrows indicate the growth of the innermost drops and paired blue arrows indicate the breakup of the innermost drop. Paired red arrows indicate the breakup at the trailing edge and paired green arrows indicate the breakup at the leading edge (color figure online)

templates to produce polymersomes. The satellite drops are easily removed by exploiting their density which becomes lower than that of the continuous phase as chloroform quickly evaporates; by contrast, polymersomes have higher density than the continuous phase.

When the sum of $Q_{1}$ and $Q_{2}$ is insufficiently large at the same value of $Q_{3}$, the inner and middle phases produce a widening coaxial jet that forms through the orifice of the collection capillary as denoted with inverted triangles in Fig. 2a, b (Utada et al. 2007). This produces more polydisperse double-emulsion drops with ill-controlled size; moreover, the number of inner drops is also highly variable. In this case, the inner interface first breaks into drops, which are then encapsulated into double-emulsion drops upon breakup of the outer interface. When $Q_{2}$ is too small by comparison with $Q_{1}$, the middle stream cannot shield the aqueous inner stream, resulting in leakage of the innermost stream into the continuous phase without generation of double-emulsion drops, as denoted with crosses $(x)$ in Fig. 2a.

For a wide range of $Q_{3}$, innermost-drop-triggered breakup of the middle phase also occurs; this is denoted with circles and squares for equal value of $Q_{1}$ and $Q_{2}$ in Fig. 4. For higher values of $Q_{1}, Q_{2}$, and $Q_{3}$, a widening jet forms and then breaks into polydisperse double-emulsion drops in the same fashion as for the widening jet in Fig. 2. This is denoted with inverted triangles in Fig. 4. By contrast, if $Q_{1}$ and $Q_{2}$ remain large, but $Q_{3}$ is reduced, a widening jet of the middle phase is formed and contains monodisperse innermost drops, as denoted with diamonds. Although the innermost drops are generated in the dripping mode (Umbanhowar et al. 2000), they cannot trigger the breakup of the middle phase jet due to the formation of the thick jet and the resultant proximity of the wall of the collection capillary (Guillot et al. 2007). Instead, the breakup of the jet occurs downstream, resulting in large double-emulsion drops containing several tens of the innermost drops. For a narrow range of low $Q_{1}$, low $Q_{2}$, and high $Q_{3}$, we observe a different mode of the doubleemulsion drop generation as denoted with unfilled circles in Fig. 4, where inner and middle streams form a narrowing coaxial jet at the orifice of the collection capillary and then break into monodisperse, but smaller double-emulsion drops. This breakup is caused by the Plateau-Rayleigh instability of the jet (Utada et al. 2007).

\subsection{Production of polymersomes in a parallelized device}

To parallelize the production of double-emulsion drops, we inject the same innermost, middle, and continuous phases into a parallelized device consisting of four single drop makers with the total values of $Q_{1}, Q_{2}$, and $Q_{3}$ set to be $4,000,4,000$, and $12,000 \mu 1 / \mathrm{h}$. This ensures that the flow rate of each device is nearly the same as an optimum value used to produce monodisperse double-emulsion drops in a single device. However, each channel has a different hydraulic resistance due to its different position relative to the inlet needle; thus, the flow rates in each drop maker are slightly different from one another. However, the innermost-drop-triggered breakup occurs over a wide range of flow rates as seen in Figs. 2, 4. This enables robust and stable formation of the double-emulsion drops in all four devices. The generation of the double-emulsion drops in a parallelized device is shown in Fig. 5a and Video 1 in the Supplementary. From the top drop maker, average diameters of the inner drops and the outer drops in each channel are 117 and $141 \mu \mathrm{m}, 112$ and $133 \mu \mathrm{m}, 109$ and $134 \mu \mathrm{m}$, and 135 and $150 \mu \mathrm{m}$, respectively, and the coefficient of variation $(\mathrm{CV})$ of the diameters in each channel is less than $2 \%$. The innermost-drop-triggered breakup is also clearly observed in a motion taken by fast camera (Phantom V9.0) in one drop maker of the parallelized device as shown in Video 2 in the Supplementary; satellite drops are also produced from the breakup of the middle oil jet at both its leading and trailing edges. Such an innermost-drop-triggered breakup occurs in all drop makes of the parallelized device for a wide range of flow rates, providing robust and stable production of double-emulsion drops. The resultant double-emulsion drops are collected in $50 \mathrm{mM}$ aqueous solution of $\mathrm{NaCl}$ which has the same osmolarity as the 


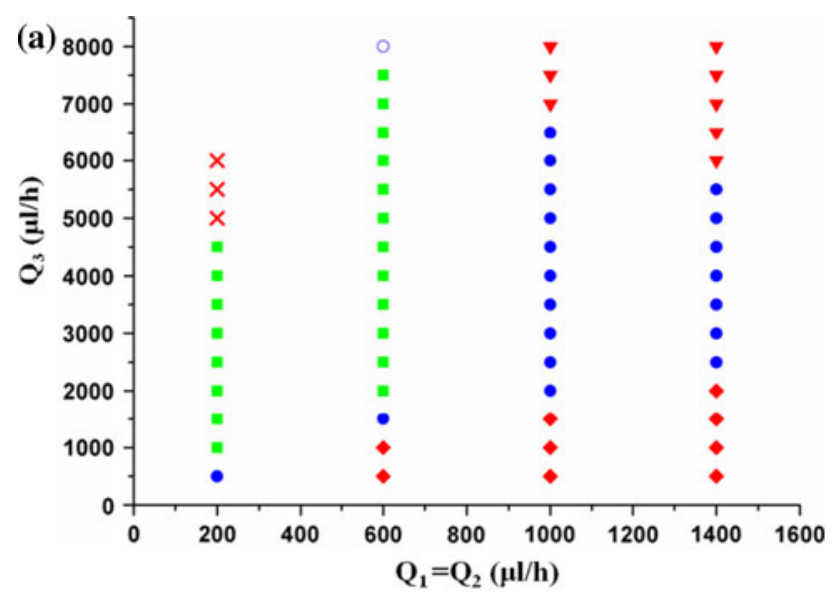

(b)

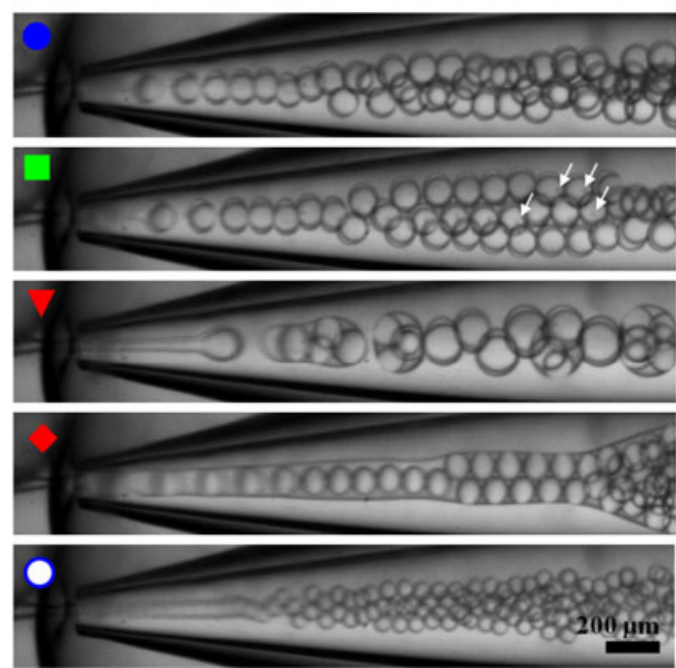

Fig. 4 Flow behavior as a function of $Q_{1}, Q_{2}$, and $Q_{3}$, where $Q_{1}$ and $Q_{2}$ are set to be equal. Blue circles and green squares denote generation of monodisperse double-emulsion without and with small satellite drops, respectively. The satellite drops are denoted with white arrows in $\mathbf{b}$. Red inverted triangles denote formation of polydisperse double-emulsion drops from a widening jet. Red diamonds denote a widening middle phase jet confining monodipserse innermost drops, which results in double-emulsion drops containing tens of the innermost drops. Unfilled blue circles denote monodisperse double-emulsion drops produced from a narrowing coaxial jet (color figure online)

innermost aqueous drops of $10 \mathrm{wt} \% \mathrm{PEG}, 100 \mathrm{mOsm} / \mathrm{L}$, but with a lower density. The double-emulsion drops are transformed into monodisperse polymersomes, consisting of a bilayer membrane of biocompatible PEG-b-PLA diblock-copolymers, through a dewetting of the middle phase from the surface of the innermost water drops, followed by subsequent separation of oil drops (Shum et al. 2011). Because the density of the innermost drop is higher than that of the collection liquid, the polymersomes are concentrated on the bottom of vial as shown in Fig. 5b. The average diameter of the resultant polymersomes, $118 \mu \mathrm{m}$, is the same as the average diameter of the inner drops of double-emulsion drops produced from four drop makers (a)

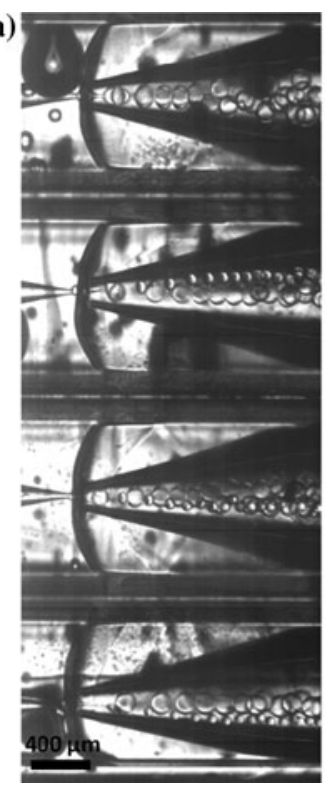

(b)

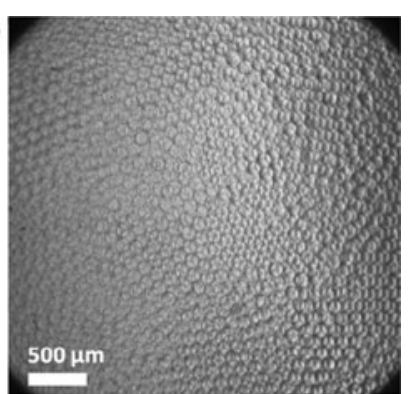

(c)

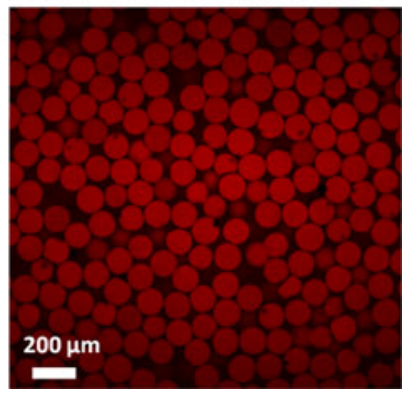

Fig. 5 a Optical microscope image of a parallelized capillary microfludic device which produces double-emulsion drops in each drop maker, simultaneously. b, c Optical and confocal microscope images of monodisperse polymersomes produced by a parallelized device (color figure online)

and the $\mathrm{CV}$ of the polymersome mixture is approximately $9 \%$. By contrast, satellites and oil drops separated from the innermost drops rise upward because their density is lower than that of the collection liquid; the volatile chloroform quickly evaporates. The production rate of the doubleemulsion drops and thus polymersomes is enhanced by a factor of approximately 4 by comparison to that of a single drop maker; for example, for polymersomes with average diameter of $118 \mu \mathrm{m}$, the total production rate is as high as $1,291 \mathrm{~s}^{-1}$ in the parallelized device, while the production rate in a single drop maker is approximately $400 \mathrm{~s}^{-1}$ at maximum for the same size of drops. The production rate can be further enhanced by optimization of flow rates in the parallelized device.

These polymersomes can encapsulate any hydrophilic molecules without loss. We demonstrate this by dissolving red dye molecules, sulforhodamine $\mathrm{B}$, in the innermost aqueous phase at a concentration of $10^{-5} \mathrm{M}$. The resultant polymersomes retain the red dye inside them as shown in the confocal microscope image of Fig. 5c.

\section{Conclusions}

We produce monodisperse polymersomes with an enhanced production rate using a parallelized capillary microfluidic device. We prepare W/O/W double-emulsion drops through innermost-drop-triggered breakup of the middle oil phase over a wide range of flow rates. This 
enables robust and stable generation of double-emulsion drops in a parallelized device. The double-emulsion drops are transformed into polymersomes through a dewetting of the middle oil phase, followed by subsequent separation of the oil from the innermost drop. Although size distribution of polymersome mixture produced from a parallelized device is wider than that from a single drop maker, the distribution is still at an acceptable level for practical usage. The size distribution can be potentially narrowed by employing the same size of all orifices and distance between the orifices. The enhancement of the production rate of biocompatible polymersomes in a parallelized capillary device and $100 \%$ encapsulation efficiency will help enable the use of the polymersomes for practical purposes, such as in encapsulation and delivery of active ingredients, including drugs, cosmetics, and nutrients. In addition, further scale up of production can be achieved through the integration of more drop makers in parallel.

Acknowledgments This work was supported by Amore-Pacific, the National Science Foundation (DMR-1006546) and the Harvard Materials Research Science and Engineering Center (DMR-0820484).

\section{References}

Anna SL, Bontoux N, Stone HA (2003) Formation of dispersions using "flow focusing" in microchannels. Appl Phys Lett 82(3): 364-366

Choi SW, Zhang Y, Xia YN (2009) Fabrication of microbeads with a controllable hollow interior and porous wall using a capillary fluidic device. Adv Funct Mater 19(18):2943-2949

Dendukuri D, Tsoi K, Hatton TA, Doyle PS (2005) Controlled synthesis of nonspherical microparticles using microfluidics. Langmuir 21(6):2113-2116

Guillot P, Colin A, Utada AS, Ajdari A (2007) Stability of a jet in confined pressure-driven biphasic flows at low Reynolds numbers. Phys Rev Lett 99(10):104502

Hwang DK, Dendukuri D, Doyle PS (2008) Microfluidic-based synthesis of non-spherical magnetic hydrogel microparticles. Lab Chip 8(10):1640-1647

Kim SH, Weitz DA (2011) One-step emulsification of multiple concentric shells with capillary microfluidic devices. Angew Chem Int Ed 50(37):8731-8734

Kim JW, Utada AS, Fernandez-Nieves A, Hu ZB, Weitz DA (2007) Fabrication of monodisperse gel shells and functional microgels in microfluidic devices. Angew Chem Int Ed 46(11):1819-1822

Kim SH, Jeon SJ, Jeong WC, Park HS, Yang SM (2008a) Optofluidic synthesis of electroresponsive photonic Janus balls with isotropic structural colors. Adv Mater 20(21):4129-4134

Kim SH, Jeon SJ, Yang SM (2008b) Optofluidic encapsulation of crystalline colloidal arrays into spherical membrane. J Am Chem Soc 130(18):6040-6046

Kim SH, Sim JY, Lim JM, Yang SM (2010) Magnetoresponsive microparticles with nanoscopic surface structures for remotecontrolled locomotion. Angew Chem Int Ed 49(22):3786-3790

Kim SH, Abbaspourrad A, Weitz DA (2011a) Amphiphilic crescentmoon-shaped microparticles formed by selective adsorption of colloids. J Am Chem Soc 133(14):5516-5524
Kim SH, Kim JW, Cho JC, Weitz DA (2011b) Double-emulsion drops with ultra-thin shells for capsule templates. Lab Chip 11(18):3162-3166

Kim SH, Shum HC, Kim JW, Cho JC, Weitz DA (2011c) Multiple polymersomes for programmed release of multiple components. J Am Chem Soc 133(38):15165-15171

Lee D, Weitz DA (2008) Double emulsion-templated nanoparticle colloidosomes with selective permeability. Adv Mater 20(18): 3498-3503

Lorenceau E, Utada AS, Link DR, Cristobal G, Joanicot M, Weitz DA (2005) Generation of polymerosomes from double-emulsions. Langmuir 21(20):9183-9186

Nie ZH, Li W, Seo M, Xu SQ, Kumacheva E (2006) Janus and ternary particles generated by microfluidic synthesis: design, synthesis, and self-assembly. J Am Chem Soc 128(29):94089412

Nisisako T, Torii T (2007) Formation of biphasic Janus droplets in a microfabricated channel for the synthesis of shape-controlled polymer microparticles. Adv Mater 19(11):1489-1493

Nisisako T, Torii T (2008) Microfluidic large-scale integration on a chip for mass production of monodisperse droplets and particles. Lab Chip 8(2):287-293

Nisisako T, Torii T, Takahashi T, Takizawa Y (2006) Synthesis of monodisperse bicolored Janus particles with electrical anisotropy using a microfluidic co-flow system. Adv Mater 18(9):11521156

Okushima S, Nisisako T, Torii T, Higuchi T (2004) Controlled production of monodisperse double emulsions by two-step droplet breakup in microfluidic devices. Langmuir 20(23): 9905-9908

Ota S, Yoshizawa S, Takeuchi S (2009) Microfluidic formation of monodisperse, cell-sized, and unilamellar vesicles. Angew Chem Int Ed 48(35):6533-6537

Romanowsky MB, Abate AR, Rotem A, Holtze C, Weitz DA (2012) High throughput production of single core double emulsions in a parallelized microfluidic device. Lab Chip 12:802-807

Shum HC, Kim JW, Weitz DA (2008) Microfluidic fabrication of monodisperse biocompatible and biodegradable polymersomes with controlled permeability. J Am Chem Soc 130(29):95439549

Shum HC, Santanach-Carreras E, Kim JW, Ehrlicher A, Bibette J, Weitz DA (2011) Dewetting-induced membrane formation by adhesion of amphiphile-laden interfaces. J Am Chem Soc 133(12):4420-4426

Thorsen T, Roberts RW, Arnold FH, Quake SR (2001) Dynamic pattern formation in a vesicle-generating microfluidic device. Phys Rev Lett 86(18):4163-4166

Umbanhowar PB, Prasad V, Weitz DA (2000) Monodisperse emulsion generation via drop break off in a coflowing stream. Langmuir 16(2):347-351

Utada AS, Lorenceau E, Link DR, Kaplan PD, Stone HA, Weitz DA (2005) Monodisperse double emulsions generated from a microcapillary device. Science 308(5721):537-541

Utada AS, Fernandez-Nieves A, Stone HA, Weitz DA (2007) Dripping to jetting transitions in coflowing liquid streams. Phys Rev Lett 99(9):094502

Xu S, Nie Z, Seo M, Lewis P, Kumacheva E, Stone HA, Garstecki P, Weibel DB, Gitlin I, Whitesides GM (2005) Generation of monodisperse particles by using microfluidics: control over size, shape, and composition. Angew Chem Int Ed 44(25):3799

Zhao YJ, Zhao XW, Sun C, Li J, Zhu R, Gu ZZ (2008) Encoded silica colloidal crystal beads as supports for potential multiplex immunoassay. Anal Chem 80(5):1598-1605 